\title{
Rasgos vocacionales de estudiantes de bachillerato aspirantes a una formación inicial docente
}

Vocational traits of senior high school students aspiring to enter initial teacher training

Traços vocacionais de alunos do ensino médio que aspiram à formação inicial de professores

\section{DOI: https://doi.org/10.18861/cied.2021.12.1.3054}

Sandra Paola Sunza Chan

Universidad Autónoma de Yucatán

México

ssunza@correo.uady.mx

ORCID: 0000-0003-1964-8571

\section{Yuliet Isabel Medina Sanguino}

Facultad de Educación, Universidad Autónoma de Yucatán

México

yuliet.medina.sanguino@gmail.com

ORCID: 0000-0001-8306-9318

Neydi Daniela Rodriguez Cenn

Facultad de Educación. Universidad Autónoma de Yucatán

México

neydi_daniela@hotmaill.com

ORCID: 0000-0002-4875-5168

Fecha de recibido: 11/12/2020

Fecha de aprobado: 22/02/2021

Cómo citar: Sunza Chan, S. P., Medina Sanguino, Y. I., \& Rodriguez Cenn, N. D. (2021). Rasgos vocacionales de estudiantes de bachillerato aspirantes a una formación inicial docente. Cuadernos de Investigación Educativa, 12(1). https://dol. org/10.18861/cled.2021.12.1.3054

\section{Resumen}

El objetivo del presente articulo es describir los rasgos vocacionales (área de especialidad, motivos, intereses y aptitudes) de en el ámbito de la educación Para ello se aplicó un estudio cuantitativo de naturaleza descriptiva y transeccional a una muestra de 252 estudiantes en fase de egreso de bachillerato, quienes manifestaron que cursar un programa enfocado en la formación inicial docente está entre sus tres primeras opciones de carrera a nivel de licenciatura. Los resultados muestran que menos de la mitad de los estudiantes cursaron un área de especialidad de bachillerato acorde con la formación docente. Por un lado se halló una tendencia a la elección de esta carrera en función de motivos externos tales como la influencia familiar y la situación economica. Por otro lado, el analisis mostro que unicamente la tercera parte de los estudiantes posee en un nivel alto los intereses vocacionales relacionados con una licenciatura en educacion, mientras que las aptitudes vocacionales requeridas para este perfí profesional se manifestaron en un nivel bajo e intermedio entre los participantes. De modo general, el estudio lleva a conclusiones Esto se debe a que muestran factores de riesgo potenciales para su futura permanencia y satisfacción vocacional en algún programa de licenciatura de este tipo pues los resultados manifiastan que los aspirantes posel los requisitos minimos par área en la que desean formarse. Esto adquiere una mayor relevancia a la luz de los retos actuales para quienes se preparan para la profesión docente, ante los cambios sociales y necesidades emergentes en los contextos escolares que añaden a este perfl nuevas y abundantes demandas

Palabras clave: formación inicial docente, elección de carrera, orientación vocacional, intereses vocacionales, aptitudes vacacionales, educación media superior

\section{Abstract}

The aim of the present article is to describe the vocational traits (area of specialization, motivation. interests and qualifications) of senior high school students from the State High School subsystem in Yucatan, Mexico, who aspire to obtain a bachelor's degree in the field of education. For such purpose, a quantitative study of a descriptive and transectional nature was carried out on a sample of 252 senior high school students in graduate phase, who stated that pursuing a plan of studies results show that less than half of the students studied a high school specialty area in accordance

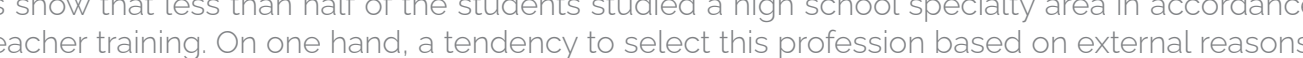
such as family influence and economic situation, was evidenced. On the other hand, the analysis showed that only a third of the students had a high level of vocational interests related to a bachelor's and intermediate level wile the vocational skills required for this professional profile showed alow conclusions about the vocational traits of senior high school students who aspire to initial teacher training. Said concusions show potential risk factors for their future permanence and vocational satisfaction in a bachelor's degree program of this type, since the results evidence that the applicants meet the minimum requirements for the area in which they wish to be trained. This becomes more elevant in light of the current chattenges for those who are getting prepared for the teaching encession, in the face of social changes and needs emerging and abundant demands to this profite.

vocational skills, high school educatio

\section{Resumo}

O objetivo deste artigo é descrever os traços vocacionais lárea de especialidade, motivos, interesses e aptidões) de alunos do ensino médio do Subsistema Estadual de Ensino Médio de Yucatán, Mexico, que pretendem cursar uma licenciatura na area de educaçao. Para tanto, foi realizado fase de conclusão do ensino médio que afirmaram que fazer um curso voltado para a formação inicial de professores está entre as três primeiras opcōes de carreira na graduação. Os resultados mostram que menos da metade dos alunos cursou alguma área de especialidade do ensino médio de acordo com a formação de professores, da mesma forma, houve uma tendência de se escolher essa carreira por motivos externos, como influência familiar e situação econômica. Por outro lado, a análise mostrou que apenas um terço dos alunos tem os interesses profissionais relacionados com uma licenciatura em ensino de nivel superior, enquanto as competências profissionais exigidas para geral, o estudo leva a conclusoes importantes sobre as caracteristicas vocacionais dos alunos do ensino medio que aspiram à formaçáo inicial de professores, uma vez que apresentam potenciais atores de risco para sua futura permanêncla e satisfaçáo profissionatem um programa de graduação aeste tipo, visto que os resultados mostram que os candidatos apresentam os requisitos minimos para quem se prepara para a profissão docente, dadas as transformacões e necessidades sociais emergentes em contextos escolares, que agregam novas e abundantes demandas a esse perfil.

Palavras-chave: formação inicial de professores, escolha de carreira, orientação profissional. nteresses profissionais, competências profissionais, ensino médió 


\section{Introducción}

El desarrollo de una carrera es un concepto ampliamente utilizado en el ámbito disciplinar de la orientación educativa y es entendido como un proceso ontogenético (Crites, 1962, como se citó en Morchio, 2009), que guarda estrecha relación con la consolidación de la personalidad de un sujeto (Holland, 1997) y que se manifiesta como una expresión de su autoconcepto (Súper y Bohn, 1953). Entre los modelos teóricos de más antigua tradición en el campo del desarrollo vocacional se encuentra la teoría de rasgo-factor formulada por Parsons (1909), en la que se plantea que cada ocupación requiere de los sujetos que la desempeñan un conjunto de características individuales para dar respuesta a las exigencias de ese campo ocupacional.

A partir de la revisión de la literatura existente en lo que respecta al estudio de este fenómeno es posible encontrar diversos elementos relevantes que han sido vinculados a los perfiles vocacionales. Entre ellos, los intereses (Gravini y Pineda, 2009; Lobato, 2002; Schilling, Gajardo y Alauf, 2019), las aptitudes (González, 2003; Lomprey, 2012; Ruperti, Gómez y Palma, 2020), Los valores (Betz y Fitzgerald, 1987. como se citó en Santos y Porto, 2002; Cortés, 2006; Vargas, 2019), los antecedentes educativos (León y Rodríguez, 2008), los motivos (Garcia, Gareka y Vázquez, 2020; Panqueba y Mesa, 2014) y los elementos contextuales (Covarrubias, 2013; Stabile et al., 2017), entre otros.

A efectos del presente trabajo se analizan cuatro aspectos considerados como rasgos vocacionales indiscutiblemente centrales en la elección de estudios enfocados en la formación inicial docente en los estudiantes de bachillerato, a saber: sus antecedentes académicos representados por el área de especialidad de bachillerato y los motivos de su elección, así como su perfil de intereses y aptitudes vocacionales.

\section{Planteamiento del problema}

Existen evidencias basadas en investigación de que una gran parte de los estudiantes que ingresan a licenciaturas de formación inicial docente lo hacen por razones que no se encuentran directamente vinculadas con sus rasgos vocacionales. Por ejemplo, García y Organista (2006) realizaron un estudio para conocer la motivación y expectativas para ingresar a la carrera de profesor de educación primaria. Los resultados mostraron que, aunque la mayor parte de los estudiantes refirieron que su incorporación a la carrera fue por interés propio, cerca del 22\% ingresó por otros motivos tales como deseos familiares, condiciones económicas y por no haber sido admitidos en la carrera de su preferencia.

Asimismo, Guzmán (2005; citado por Mungarro y Zayas, 2009) señala que entre los principales factores determinantes en la elección de estas carreras destacan el género, el nivel socioeconómico y los estereotipos profesionales.

Se han realizado estudios para conocer las causas por las cuales estudiantes que cursan carreras de formación inicial docente desertan. Al respecto, Guzmán y Romero (2006) realizaron una investigación en el estado de Baja California, México, para conocer cuáles fueron las causas que ocasionaron que un 23.3\% de los alumnos de las licenciaturas en docencia abandonaran sus estudios. Entre las principales razones destacó la insatisfacción con la carrera, la cual se vinculó directamente a deficiencias en el proceso de elección vocacional. Es necesario señalar que estas problemáticas en México no son exclusivas de los programas formativos enfocados en la docencia, ni de la educación superior. Muestra de esto son los resultados derivados de investigaciones como las de la OCDE sobre la 
deserción escolar y la eficiencia terminal, en las que se reportó que únicamente el $68 \%$ de los estudiantes de bachillerato en México terminan sus estudios, mientras que un 32\% no lo logra (Santiago, McGregor, Nusche, Ravela y Toledo, 2012), con lo cual se truncan sus aspiraciones a continuar con estudios de niveles superiores.

Debido a esto, en el Sistema de Educación Media Superior se han implementado estrategias con el fin de fortalecer el desarrollo académico, personal y vocacional del alumnado en esta etapa, así como para mejorar los procesos de toma de decisiones hacia los niveles educativos subsiguientes. Actualmente se implementa en todos los bachilleratos de México el programa Construye T el cual se enfoca en acciones para elevar el bienestar presente y futuro del alumnado, asi como en el desarrollo de habilidades para enfrentar exitosamente sus retos académicos y personales (Secretaría de Educación Pública, 2020). Una de las principales áreas de atención de esta estrategia es la vocacional, cuyo objetivo se enfoca en la construcción de un proyecto personal que permita al estudiante transitar de modo exitoso hacia los estudios superiores, tomando en cuenta sus intereses, aptitudes y motivación.

Sin embargo, desde su puesta en marcha en 2007 hasta la fecha, las problemáticas de deserción vinculada a factores de desarrollo vocacional en estudiantes que ya han ingresado a instituciones de educación superior se mantienen vigentes. Esto permite ver que es necesario continuar con la investigación en torno a este fenómeno y con el diseño de estrategias que coadyuven a reducir su impacto.

En el caso específico de los programas de formación inicial docente, además de los elementos ligados al proceso de elección vocacional es posible señalar que los mecanismos de ingreso, aunque difieren de una institución de educación superior a otra y en función de las políticas imperantes en cada país, se basan en una evaluación general de habilidades académicas, sin considerar los rasgos vocacionales del aspirante. De acuerdo con Barber \& Mourshed (2007), en España los sistemas educativos cuentan con mecanismos más eficientes para seleccionar a los aspirantes a dichos programas del área educativa. Esto parte de la idea de que un estudiante que aspira a formarse y convertirse en un docente eficiente debe poseer cierto conjunto de caracteristicas susceptibles de identificación antes de ejercer la profesión (Aguirre, Dévora y Valenzuela, 2015).

En contraste, en México, los mecanismos de ingreso a las universidades son generales y no existen métodos para identificar si los candidatos a ingresar a esta área son idóneos para formarse en el programa de estudio. Ibáñez (1999, citado por Martín, 2015), señala que en este país el método de selección de aspirantes para ingresar a algún programa educativo se basa en algo tradicional, que consiste en la aplicación de un examen de habilidades generales en donde se da importancia a los puntajes que se obtienen. De este modo, la manera de evaluar no considera otros factores que puedan influir para determinar a los mejores candidatos para los programas educativos, y se deja de lado la consideración de que un puntaje alto en un examen de habilidades generales no es un predictor de permanencia y satisfacción vocacional.

Esto adquiere una mayor relevancia cuando se enfoca en la profesión docente, alser una de las carreras con mayores demandas derivadas de las necesidades sociales presentes en los contextos educativos, lo que hace de este perfil profesional uno de los más complejos en lo que respecta a las competencias necesarias para un desempeño laboral eficaz. Ante tal circunstancia resulta indispensable realizar un análisis de los rasgos vocacionales de aquellos estudiantes de bachillerato que expresan su aspiración a continuar sus estudios superiores en este ámbito, a fin de identificar si cuentan con las caracteristicas pertinentes para desempeñarse de modo competente en esta área. 


\section{Marco teórico}

\section{Componentes del desarrollo de la vocación}

La orientación vocacional es un proceso cuyo propósito es orientar al individuo en la toma de decisiones, considerando diferentes componentes que, interrelacionados, dan como origen la elección de una carrera (Morales, 2017). Algunos de los más relevantes se abordarán en este apartado.

En primer lugar se señala la importancia de los antecedentes educativos, los que hacen referencia a la formación recibida a lo largo de la vida y, a efectos del presente trabajo, específicamente el conjunto de conocimientos, habilidades y actitudes que el estudiante adquiere durante el bachillerato. En México, estos se encuentran establecidos en el Marco Curricular Común, uno de los pilares de la Reforma Integral de Educación Media Superior (RIEMS). De tal modo se espera que el estudiante realice una elección vocacional congruente con el área de especialidad que curse durante su formación en dicho nivel.

En segundo lugar es necesario hacer hincapié en los motivos de elección vocacional, término que se refiere al impulso que conduce a una persona a realizar una determinada acción o a seguir una dirección para desarrollarse tanto personal como académicamente. La motivación es uno de los factores principales que conducen a los sujetos a hacer una elección de carrera. Asimismo, el motivo de elección se relaciona con las necesidades de crecimiento y autorrealización, como un deseo humano de buscar el desarrollo de sus potencialidades (Garcia, Gareka y Vázquez, 2020; Mungarro y Zayas, 2009). De este modo puede señalarse que los motivos de elección vocacional se relacionan con el desarrollo de la identidad de los sujetos, siendo la base de la toma de decisiones hacia el futuro.

En tercer lugar, entre los componentes más importantes de la elección vocacional se encuentran los intereses debido a que, si son tomados en cuenta, contribuyen de manera positiva a que el proceso de desarrollo vocacional tenga mayores probabilidades de éxito. En este sentido, Lent, Brown y Hackett (1994, citados en Foligato, Pérez, Olaz y Parodi, 2003, p. 61), definen el interés vocacional como "patrones de gustos, indiferencias y aversiones respecto a actividades relacionadas con carreras y ocupaciones". El interés se manifiesta cuando se experimenta rechazo o se siente atracción por ciertas ocupaciones. El hecho de que el sujeto sea consciente de las inclinaciones o aspiraciones de estudio que tiene a futuro lo guiará para realizar el diseño de su proyecto de vida académica, optar por una elección vocacional acorde a su perfil, ampliar sus expectativas y orientarlo en la toma de decisiones (Schilling, Gajardo y Alauf, 2019). De este modo es posible garantizar menores riesgos de abandono o deserción escolar (Lobato, 2002).

Finalmente se encuentran las aptitudes, es decir, las capacidades y destrezas que utiliza un individuo para desenvolverse de manera satisfactoria en actividades determinadas. La Real Academia Española (RAE, 2019) las define como la "capacidad para operar competentemente en una determinada actividad" (Def. 1). En la misma linea, Lomprey (2012) define aptitud como "la capacidad potencial del individuo para efectuar una actividad o un trabajo" (p.34). La aptitud, entonces, puede ser considerada como uno de los componentes vocacionales más relevantes en el proceso de selección de una carrera u ocupación (González, 2003: Ruperti, Gómez y Palma, 2020). 


\section{Elección vocacional en carreras de formación inicial docente}

Como se ha analizado en el apartado anterior, la elección de una carrera se encuentra influida por diferentes factores. Sin embargo, se espera que los elementos predominantes sean de naturaleza intrinseca y se encuentren vinculados de modo directo a las preferencias y rasgos del sujeto. Cuando se habla de docencia es necesario poner especial atención debido a las responsabilidades y compromisos que conforman este perfil profesional en el contexto escolar. Denzler y Wolker (2009, citados por Gratacós, 2014) mencionan que "la importancia de tener buenos profesores para garantizar la calidad del sistema educativo depende, en gran parte, de quien opta por una carrera docente" (p.5). A pesar de esto, Sánchez (2003) menciona que:

- Debemos entender que la vocación hacia el magisterio puede ocupar un lugar destacado entre algunos aspirantes, aunque, en otros, dicha inclinación se puede ver anulada por factores de orden económico o académico, que solapan su verdadero interés. Por tanto, no todos los que acceden a las Escuelas de Magisterio lo hacen movidos por un sentido vocacional, ya que existen otras circunstancias en dicho acceso, que han llegado a tener un mayor peso específico. (p.214)

Con esto se entiende que existen dos grandes grupos de personas que eligen la profesión docente. El primer grupo incluye a los aspirantes que toman esta decisión por vocación, es decir, aquellos que poseen los intereses, las habilidades, los antecedentes académicos y la motivación requeridos para formarse en dicha área. El segundo grupo, sin embargo, elige por otras razones, sin estar seguros de querer formarse para esta función. En este último caso, algunas de las consecuencias asociadas pueden ser la deserción escolar o la insatisfacción con la carrera, lo que incide en las posibilidades de lograr un desempeño exitoso en el ejercicio de la profesión.

En este sentido García (2010) coincide en que la profesión docente es uno de los perfiles que exige dominar un conjunto de conocimientos y competencias al ingresar a los estudios de nivel superior. De lo contrario existen probabilidades de fracaso escolar y, a su vez, a largo plazo, en el ámbito profesional.

La idea anterior corrobora la importancia de los rasgos vocacionales y, especificamente, de los antecedentes académicos en los aspirantes a licenciaturas de este tipo. En la misma línea, autores como López (2018) recomiendan que los aspirantes a la formación inicial docente cubran ciertas competencias y campos disciplinares, especialmente en lo referente a las ciencias sociales y humanidades, con el fin de poseer bases sólidas para la comprensión del comportamiento humano como punto de partida para la adquisición de las habilidades requeridas para la profesión, las que se fortalecerán durante el trayecto formativo de nivel superior.

En sintesis, es indispensable insistir en la importancia de que los aspirantes a una formación inicial docente cuenten con los rasgos vocacionales para dicha área, así como con una congruencia entre esta aspiración y su proyecto de vida personal y profesional. Esto incrementará las posibilidades de que los procesos formativos desemboquen en el egreso de profesionales competentes para dar respuesta a las numerosas necesidades presentes en los contextos educativos actuales.

A partir de lo expuesto es posible afirmar que la formación de docentes es un tópico de investigación e intervención prioritarias puesto que el rol del profesorado exige una transformación vinculada a los cambios y problemáticas sociales de la actualidad. Frente a estos retos resulta necesario dirigir la mirada e indagar sobre 
los rasgos vocacionales de los aspirantes a licenciaturas del campo educativo bajo la premisa de que esto puede establecer las bases para emprender acciones hacia la formación de profesionales mejor capacitados.

\section{Diseño y metodología}

\section{Tipo de estudio}

La presente investigación forma parte del proyecto "Percepciones sobre la demanda de las Licenciaturas de la Facultad de Educación, de la Universidad Autónoma de Yucatán", desarrollado con el fin de indagar sobre las preferencias e intereses de los estudiantes de bachillerato que desean estudiar una licenciatura en el área de la educación.

A partir de la finalidad de dicho proyecto se estableció el objetivo especifico de la investigación en la que se centra este artículo y que es el de describir los rasgos vocacionales (área de especialidad, motivos, intereses y aptitudes) de estudiantes de bachillerato del subsistema de Preparatorias Estatales en Yucatán, México, que aspiran a estudiar una licenciatura en el ámbito de la educación.

Para dar respuesta a este objetivo se realizó un estudio descriptivo de carácter cuantitativo. El diseño de la investigación fue no experimental pues no existió manipulación en las distintas variables que se presentaron en el instrumento (Rojas, 2005), sino que se estudiaron tal como se presentaron en los participantes. En lo referente a su temporalidad, este se clasifica como transeccional puesto que se recolectaron los datos en un solo momento (Hernández, Fernández y Baptista, 2010).

\section{Participantes}

Los participantes en esta investigación fueron los estudiantes de tercer año de ocho escuelas preparatorias estatales de Yucatán. Se encuestó a un total de 1171 alumnos de este subsistema de bachillerato. Sin embargo, a efectos del presente trabajo, la muestra estuvo conformada por los 252 sujetos que seleccionaron entre sus tres primeras opciones vocacionales la aspiración de optar por una formación inicial docente.

\section{Instrumento}

A fin de responder al objetivo del proyecto de investigación, se construyó el instrumento denominado "Cuestionario de Percepciones sobre la demanda de las Licenciaturas de la Facultad de Educación, UADY", a partir de un proceso de trabajo colaborativo entre los investigadores participantes, tomando como base instrumentos institucionales previamente construidos e incorporando los principios implicitos en el Cuestionario sobre intereses y aptitudes vocacionales de Luis Herrera y Montes (Villegas, 2009) sobre los rasgos vinculados con el perfil del profesional de la educación. El instrumento resultante se compone de cinco secciones: datos generales, intereses generales, intereses en el área educativa, intereses vocacionales y aptitudes vocacionales. 
El instrumento fue validado por jueces expertos, basado en los siguientes criterios: claridad, coherencia, relevancia y suficiencia del instrumento. A efectos del presente trabajo se analizaron los resultados concernientes a los reactivos de antecedentes académicos y motivos de elección de carrera correspondientes a la sección de intereses generales, así como los apartados de intereses y aptitudes vocacionales. Para estos dos últimos se calculó el Alfa de Cronbach, obteniendo una validez mayor a 0.80 , con lo cual se concluye que su fiabilidad es alta.

\section{Procedimiento para el análisis de datos}

Se construyó una base de datos en la versión 18 del Statistical Package for the Social Sciences (SPSS), utilizando procesos de estadistica descriptiva a fin de obtener los resultados que se presentan en el siguiente apartado.

\section{Resultados y discusión}

En este apartado se analizan los principales resultados obtenidos en torno al objetivo planteado para el estudio, por lo que se describen los hallazgos en función de cada uno de los rasgos vocacionales considerados en este trabajo, a saber: antecedentes académicos (área de especialidad en bachillerato), motivos, intereses y aptitudes vocacionales.

Tal como ya se mencionó, la muestra estuvo constituida por estudiantes de ocho escuelas preparatorias estatales, quienes aspiran a una licenciatura relacionada con el ámbito de la educación. Un total de 252 alumnos cumplió con este criterio. Poco más de un tercio de los participantes señalaron a la formación inicial docente como su primera opción de carrera, mientras que el resto de los estudiantes la consideraron como segunda o tercera opción. En otras palabras, la mayoría de los sujetos que aspiran a cursar una licenciatura en el ámbito de la educación la consideran como una opción alternativa en caso de no poder ingresar a la carrera de su preferencia. Con respecto a esto, Mungarro (2007) opina que "la libertad del joven para elegir su carrera se ve limitada, pues suele elegir según las posibilidades de su ámbito, ajenas en muchos de los casos a sus intereses individuales". (p.34)

\section{Antecedentes académicos}

Para indagar sobre los antecedentes académicos de los aspirantes se tomó como base el área de especialidad de bachillerato que cursaron, a fin de identificar su afinidad con el ámbito de la formación inicial docente. De tal forma se procedió con base en el Marco Curricular Común, que constituye uno de los pilares de la Reforma Integral de Educación Media Superior (RIEMS), cuya finalidad es establecer el perfil del egresado de este nivel educativo, es decir, los conocimientos, habilidades y actitudes que todos los estudiantes de bachillerato deben desarrollar sin importar el subsistema al cual pertenezcan. En dicho marco se detallan las áreas de especialización de estudio en función de la modalidad de bachillerato que se curse.

A efectos del presente estudio dichas áreas se tipificaron en seis opciones, tal como se señala a continuación: 1. Ciencias biológicas, 2. Ciencias exactas, 3. Humanidades, 4. Ciencias sociales, 5. Socioeconómicas, y 6. Otros.

En la Tabla 1 se muestran los resultados obtenidos con respecto a las áreas de especialidad de bachillerato cursadas por los participantes. 
Tabla 1. Análisis de las áreas de bachillerato

\begin{tabular}{|l|c|c|}
\hline Especialidad de bachillerato & Frecuencia & Porcentaje \\
\hline Ciencias sociales & 111 & $44.8 \%$ \\
\hline Ciencias biológicas & 77 & $30.6 \%$ \\
\hline Socioeconómicas & 33 & $12.7 \%$ \\
\hline Ciencias exactas & 20 & $7.9 \%$ \\
\hline Humanidades & 11 & $4.0 \%$ \\
\hline Total & 252 & $100 \%$ \\
\hline
\end{tabular}

Fuente: Elaboración propia

Para la interpretación de los resultados se tomaron como base los planteamientos del Marco Curricular Común para determinar que las áreas de especialidad de Ciencias sociales y Humanidades tienen relación directa con las licenciaturas del área educativa, idea que también es corroborada por algunos autores ya referidos en apartados anteriores como López (2018). Así, puede notarse en la tabla 1, que 122 participantes (48.8\%) cuentan con estos antecedentes académicos. Desde una perspectiva general, esto significa que aproximadamente la mitad de los estudiantes que aspiran a la formación inicial docente si cursaron en el bachillerato un área de especialidad afín a la misma, mientras que los otros 138 (51.2\%) se formaron en otras especialidades que no guardan relación con el área de la educación, entre ellas Ciencias biológicas con un 30.6\% (77) que es la segunda con mayor cantidad de estudiantes. Esto resulta un dato interesante ya que la especialización en ciencias biológicas se relaciona más con el área de la salud, a carreras como enfermería, medicina o nutrición, entre otras.

En este sentido se identificó que una gran parte de los estudiantes que aspiran a una licenciatura en el ámbito de la educación no muestran coherencia en su proyecto de carrera, lo cual se hace evidente a partir de la falta de relación entre el perfil de especialidad cursado en bachillerato y sus opciones de educación superior. Esto cobra mayor sentido a la luz de los planteamientos que enfatizan en la importancia de la integración de todos estos elementos para incrementar las posibilidades de éxito en la toma de decisiones vocacionales. Al respecto, Parsons (1909), uno de los teóricos clásicos de la orientación educativa, propone que el individuo debe contemplar tres factores fundamentales al momento de elegir una carrera profesional. En primer lugar, el individuo debe conocerse a si mismo, tener claridad con respecto a sus habilidades, intereses, ambiciones y limitaciones. Como segundo punto el individuo debe saber las exigencias y oportunidades que existen en el mundo laboral respecto a la carrera que desea elegir $y$, finalmente, debe relacionar estos dos elementos para asegurarse de que la elección que hará sea la adecuada. A pesar de esto, en un grupo significativo de los participantes de este estudio no se refleja una relación entre su área de bachillerato y las opciones de estudio de licenciatura, representando esto un potencial riesgo en su futuro desarrollo académico y profesional.

\section{Motivos de elección vocacional de los aspirantes}

El segundo objetivo de esta investigación consistió en describir los motivos por los cuales los estudiantes de bachillerato aspiran a estudiar una licenciatura en el ámbito de la formación inicial docente. Para esto se interrogó a los estudiantes al respecto, a partir de opciones de respuestas preestablecidas, con la posibilidad de elegir más de una o agregar motivos adicionales. 
Las respuestas giraron en torno a la situación económica, las habilidades en el área, la cercanía o fácil acceso, la influencia familiar y la posibilidad de permanecer cerca de sus amigos. En la Tabla 2 se muestran las frecuencias con las que fueron señalados los distintos motivos considerados en este estudio.

Tabla 2. Análisis de motivos de elección

\begin{tabular}{|l|c|}
\hline \multicolumn{1}{|c|}{ Motivos } & f \\
\hline Habilidades en el área & 292 \\
\hline Intereses en el área & 259 \\
\hline Influencia familiar & 117 \\
\hline Situación económica & 114 \\
\hline Cercanía de tu casa & 60 \\
\hline Para estar con amigos & 9 \\
\hline Otros & 4 \\
\hline
\end{tabular}

Fuente: Elaboración propia.

Como se muestra en la Tabla 2, los motivos más frecuentes por los cuales los sujetos aspiran a estudiar una licenciatura relacionada con la educación se centran en que consideran poseer las habilidades e intereses para desarrollarse adecuadamente en el área. Sin embargo puede observarse que están presentes otros motivos tales como la influencia familiar y la situación económica. Los motivos menos frecuentes fueron la cercanía a la casa, para estar con amigos y otros. Entre estos últimos motivos resaltan aquellos vinculados con la necesidad de ingresos económicos, la consideración de la docencia como un pasatiempo, la percepción de la formación inicial docente como una carrera fácil y el hecho de no haber ingresado a la universidad de su preferencia.

Así, aunque de manera general puede observarse que los dos motivos más señalados (intereses y aptitudes) son rasgos vocacionales fundamentales para la elección de carrera, existe un número considerable de aspirantes que señaló otros motivos que pueden ser catalogados como de naturaleza extrínseca. Este hallazgo coincide con los resultados encontrados por García-Poyato, Cordero y Jiménez (2017) y por García-Poyato, Cordero y Torres (2018) en el contexto de Escuelas Normales en México.

También puede verse que estos resultados concuerdan con las ideas de algunos autores en lo referente a la existencia de variables que pueden explicar la diferenciación en las elecciones de las carreras. En primer lugar se encuentran los factores ambientales, que toman en cuenta el estatus socioeconómico, modelos de rol, estereotipos de género, entre otros. En segundo lugar los factores personales, dentro de los cuales se encuentran los intereses, expectativas de autoeficacia, autoconcepto sexual, aspiraciones y valores (Betz y Fitzgerald, 1987; citados por Santos y Porto, 2002). 


\section{Intereses de los aspirantes}

El tercer objetivo consistió en determinar en qué grado los bachilleres que aspiran a estudiar una licenciatura enfocada en la formación inicial docente poseen los intereses vocacionales vinculados a este perfil.

En cuanto al interés, puede definirse como "una actividad interna del individuo, de su iniciativa para realizar cierta actividad y de una correspondencia entre la inclinación a la actividad y la eficiencia en su ejecución" (Gravini y Pineda, 2009, p.114).

Para lograr dicho objetivo, a través de una escala tipo Likert, los sujetos señalaron su grado de interés por ciertas actividades relacionadas de modo general con el ámbito docente. Entre ellas se incluyeron situaciones vinculadas a la expresión oral y escrita, el interés por el ejercicio del liderazgo y la interacción social así como actividades directamente relacionadas con el quehacer del profesional de la educación tales como el diseño de estrategias de enseñanza-aprendizaje y de materiales educativos, entre otros.

A partir de la valoración otorgada por los participantes se establecieron rangos y se calcularon variables, analizando los resultados con base en tres niveles, con el objetivo de agrupar las respuestas. En la Tabla 3 se presentan los resultados con respecto al nivel en que los estudiantes poseen los intereses del perfil requerido para la formación inicial docente.

Tabla 3. Análisis de los intereses

\begin{tabular}{|c|c|c|c|}
\hline Bajo & \multicolumn{2}{|c|}{ Intermedio } & Alto \\
\hline $0-25$ & $26-50$ & $51-75$ & $76-100$ \\
\hline $.79 \%$ & $9.12 \%$ & $54.36 \%$ & $35.71 \%$ \\
\hline
\end{tabular}

Fuente: Elaboración propia.

Como puede observarse en la Tabla 3, la mayoría de los sujetos, es decir, el 63.48\% se ubica en un nivel intermedio, lo que significa que un poco más de la mitad considera poseer medianamente los intereses correspondientes al ámbito de la formación inicial docente. Sin embargo, únicamente el 35.71\% de los estudiantes poseen tales intereses en un nivel elevado.

Estos resultados muestran que un porcentaje significativo de estudiantes aspiran a esta carrera sin poseer niveles altos de interés vocacional en este ámbito. Esto lleva a la reflexión de que elegir una licenciatura que no va de acuerdo con las inclinaciones y preferencias personales puede afectar al estudiante, lo que se vincula a un bajo rendimiento e insatisfacción con la carrera (Lobato, 2002; Schilling, Gajardo y Alauf, 2019). A largo plazo esta problemática puede afectar el desempeño laboral de quienes finalizan una licenciatura que no está de acuerdo con sus caracteristicas individuales. Esto adquiere una fuerte relevancia en el ámbito del desarrollo profesional docente al ser un área que plantea muchos retos a quienes ejercen esta labor, debido a la complejidad en las demandas de los contextos educativos actuales, los que requieren un perfil con alta motivación en el desarrollo de estas funciones. 


\section{Aptitudes de los aspirantes}

El cuarto y último objetivo consistió en determinar en qué grado los bachilleres que aspiran a estudiar una licenciatura orientada a la formación inicial docente poseen las aptitudes vocacionales vinculadas a este perfil.

Las aptitudes son definidas de modo tradicional por Osipow (1976), como la capacidad que tienen las personas para desempeñarse exitosamente dentro de una variedad de ocupaciones o actividades especificas. En este sentido se interrogó a los participantes sobre qué tan aptos se consideran para diversas actividades vinculadas con el área de la educación.

Entre las actividades incluidas en este apartado del instrumento resaltan aquellas que demandan habilidades sociales, aptitudes para dirigir grupos, capacidades para la comunicación de ideas a través de diversos medios y habilidades de investigación, entre otras.

De modo similar al análisis presentado en el apartado anterior, a partir de la valoración otorgada por los participantes se establecieron rangos y se calcularon variables, analizando los resultados con base en tres niveles, a fin de agrupar las respuestas. Los resultados se muestran en la Tabla 4.

Tabla 4. Análisis de aptitudes

\begin{tabular}{|c|c|c|c|}
\hline Bajo & \multicolumn{2}{|c|}{ Intermedio } & Alto \\
\hline $0-25$ & $26-50$ & $51-75$ & $76-100$ \\
\hline $2.3 \%$ & $33 \%$ & $64.7 \%$ & 0 \\
\hline
\end{tabular}

Fuente: Elaboración propia.

Como puede observarse, las aptitudes vinculadas con la formación inicial docente que los participantes consideraron poseer los ubican en los niveles intermedio (97.7\%) y bajo (2.3\%). Ningún participante consideró poseer un nivel alto con respecto a las aptitudes necesarias para la docencia.

En este sentido puede decirse que los aspirantes no muestran un perfil de habilidades óptimo para desempeñarse en el ámbito de una licenciatura orientada a la formación inicial docente. Esto contrasta con las ideas de González (2003) y Ruperti, Gómez y Palma (2020) quienes señalan que las aptitudes o habilidades deben ser determinantes en los procesos de elección vocacional puesto que las mismas son indicadores del desempeño exitoso en una ocupación. Aunque este rasgo debe ser uno de los más importantes a considerar al momento de elegir una licenciatura, puede notarse que un porcentaje significativo de los bachilleres participantes no han llevado a cabo un análisis pertinente de las aptitudes que poseen ante su aspiración a un perfil profesional docente. 


\section{Conclusiones}

Con base en los resultados del presente estudio es posible concluir que las áreas de especialización de bachillerato predominantes entre los estudiantes del Subsistema de Preparatorias Estatales de Yucatán que aspiran a estudiar una licenciatura enfocada en la formación inicial docente fueron las ciencias sociales y humanidades, que representan antecedentes académicos afines a sus aspiraciones profesionales. Sin embargo, un sector significativo de los participantes cursó especialidades totalmente desvinculadas de este ámbito, lo cual muestra que no existe articulación entre distintos aspectos de su proyecto de vida. Esto evidencia la existencia de un área de oportunidad para la atención prioritaria a través de los servicios de orientación educativa que se brindan en el bachillerato. En lo referente a los motivos por los cuales los estudiantes participantes aspiran a estudiar una licenciatura en el ámbito de la educación, se identificó que los más frecuentes se centran en que consideran poseer las habilidades e intereses pertinentes. A pesar de esto puede notarse que están presentes otros motivos tales como la influencia familiar y la situación económica. Estos resultados concuerdan con algunos de los estudios referidos en los epigrafes del trabajo, como los hallazgos de García y Organista (2006) sobre la influencia de los deseos familiares, condiciones económicas y el fracaso en los intentos de ingresar a otra carrera. Concuerdan, asimismo, con el estudio de Guzmán (2005; citado por Mungarro y Zayas, 2009) en que los principales factores determinantes de la elección de estas carreras son de naturaleza extrínseca.

En cuanto al grado en que los participantes consideraron poseer los intereses vocacionales vinculados a este perfil, se identificó que la mayoría se ubica en un nivel intermedio y poco más de un tercio en el nivel alto.

En el caso de las aptitudes vocacionales orientadas al ámbito de la formación docente se encontró que los participantes manifiestan poseerlas en un nivel intermedio y bajo. En relación con esto, autores como González (2003) y Schilling, Gajardo y Alauf (2019) enfatizan en que las aptitudes o habilidades deben ser determinantes en el proceso de elección vocacional pues son indicador de adaptación y competencia para desempeñarse de modo eficaz en determinadas ocupaciones.

De modo general puede señalarse que los estudiantes que participaron en esta investigación poseen parcialmente los rasgos vocacionales vinculados con las carreras de formación docente. Se identificaron importantes áreas a mejorar en lo referente a los antecedentes académicos y aptitudes, las cuales muestran que resulta relevante emprender acciones desde los servicios de orientación y apoyo al estudiante en los bachilleratos, asi como en lo referente a los mecanismos de reclutamiento y admisión a los programas de licenciatura enfocados en este ámbito, de modo que se pueda fortalecer el perfil de ingreso de los estudiantes, incrementar sus posibilidades de permanencia y éxito escolar, así como asegurar mejores resultados en los procesos de formación de profesionales competentes para hacer frente a las problemáticas educativas emergentes en la actualidad.

\section{Limitaciones}

Entre las principales limitaciones presentes en esta investigación, por un lado puede señalarse que la diversidad existente en los subsistemas de bachillerato en México dificulta homologar metodologías de investigación y análisis, por lo cual fue necesario enfocar el estudio en un único subsistema, el de Preparatorias Estatales, por lo cual no es posible generalizar los resultados obtenidos a los otros subsistemas. 
Por otro lado, el hecho de que esta investigación formara parte de un proyecto más amplio delimitó el tiempo y acceso a las instituciones educativas, lo cual requirió la adaptación a un calendario específico.

A partir de esto es necesario señalar la recomendación de extender el estudio a los otros subsistemas de bachillerato a fin de identificar puntos en común y diferencias con los resultados expuestos en este trabajo. Asimismo, se considera importante abrir líneas de investigación que permitan indagar en otros rasgos vocacionales y diversificar las metodologias y agentes a fin de obtener resultados más integradores que aporten a la comprensión de este objeto de estudio.

\section{Nota:}

Aprobación final del artículo, editora responsable Mag. Verónica Zorrilla de San Martín.

Contribución de autoria: Las autoras han colaborado en partes iguales, en todas las etapas del artículo. 


\section{Bibliografía}

AGUIRRE, F., DÉVORA, C., Valenzuela, E. (2015). Las condiciones de ingreso a la profesión docente: un factor para el logro de la calidad educativa. Revista Electrónica de Investigación Educativa, 11, 405-412. https://Www.redalyc.org/ $\mathrm{pdf} / 461 / 46142596029 . \mathrm{pdf}$

BARBER, M., \& MOURSHED, M. (2007). How the world's best-performing school systems come out on top. ICómo se destacan los sistemas escolares con mejor rendimiento del mundo]. London: McKinsey \& Company.

CORTÉS, P. (2006)Valoresyorientación profesional:algunas líneas de investigacióne intervención actuales. Contextos Educativos, 8-9, 233-248. https://publicaciones. unirioja.es/ojs/index.php/contextos/article/download/566/530

COVARRUBIAS MÉNDEZ, L. (2013). Elección de carrera: aspectos que influyen en los jóvenes [Tesis de grado, Universidad Pedagógica Nacional Unidad Ajusco]. http://200.23.113.51/pdf/29909.pdf

FOLIGATO, H., PÉREZ, E., OLAZ, F. Y PARODI, L. (2003). Cuestionario de Intereses Profesionales Revisado. (CIP-R). Análisis de sus Propiedades Psicométricas. Laboratorio de Evaluación Psicológica y Educativa, 3, 61-79. https://core.ac.uk/ download/pdf/334391335.pdf

GARCÍA, E. (2010). Competencias éticas del profesor y calidad de la educación. Revista electrónica interuniversitaria de formación del profesorado, 13(4) (Ejemplar dedicado a: La profesión docente: Escenarios, perfiles y tendencias) 29-42. https://dialnet.unirioja.es/servlet/articulo?codigo=3675461

GARCÍA, M., GAREKA, J. R. Y VÁZQUEZ, B. (2020). Motivación adolescente y educación superior. Revista científica multidisciplinar UNIFLU. 5(1), 34-52.

http://Www.revistas.uniflu.edu.br:8088/seer/ojs3.0.2/index.php/multidisciplinar/ article/view/277/170

GARCÍA, J. Y ORGANISTA, J. (2006). Motivación y expectativas para ingresar a la carrera de profesor de educación primaria: un estudio de tres generaciones de estudiantes normalistas mexicanos de primer ingreso. Revista Electrónica de Investigación Educativa, 8, 2-17. https://www.redalyc.org/pdf/155/15508208.pdf

GARCÍA-POYATO, J., CORDERO, G. Y JIMÉNEZ, J. (2017). ¿Por qué quiero ser profesor? Estudio exploratorio de las motivaciones de estudiantes normalistas de Baja California. Congreso Nacional de Investigación Educativa. http://comie. org.mx/congreso/memoriaelectronica/v14/doc/1654.pdf

GARCÍA-POYATO, J., CORDERO, G. Y TORRES, R. (2018). Motivaciones para ingresar a la formación docente. Revisión de estudios empíricos publicados en el siglo XXI. Instituto de Investigación y Desarrollo Educativo, 57, 1-26. DOl: https://dx.doi. org/10.4151/07189729-vol.57-iss.2-art.727

GONZÁLEZ, V. (2003). La orientación profesional desde la perspectiva históricocultural del desarrollo humano. Revista cubana de psicología, 20, 260-268. http://pepsic.bvsalud.org/pdf/rcp/v2on3/12.pdf

GRATACÓS, G. (2014). Estudio sobre las motivaciones en la elección de ser maestros [Tesis doctoral no publicada]. Universidad Internacional de Catalunya. https:// www.tesisenred.net/bitstream/handle/10803/134986/Tesi_Gloria_Gratacos_ Casacuberta.pdf? sequence $=1$ 
GRAVINI, M. Y PINEDA, W. (2009). Intereses profesionales de estudiantes de secundaria de la ciudad de Barranquilla. Psicogente, 12, 111-123. http://www. redalyc.org/pdf/4975/497552353009.pdf

GUZMÁN, M., ROMERO, B. (2006). Causas de deserción en las licenciaturas de docencia y traducción del idioma inglés y mecanismos de prevención 2002-I 2005-II. Universidad Autónoma de Baja California. http://idiomas.ens.uabc.mx/ plurilinkgua/docs/v2/2/GuzmanCausasDesert-espCorr.pdf

HERNÁNDEZ, R., FERNÁNDEZ, C. Y BAPTISTA, P. (2010). Metodología de la investigación. McGraw-Hill.

https://institutoprofesionalmr.org/wp-content/uploads/2018/04/ Hern\%C3\%A1ndez-Fern\%C3\%A1ndez-Baptista-2010-Metodologia-de-laInvestigacion-5ta-edicion.pdf

HOLLAND, J. (1997). La elección vocacional. Teoria de la carrera. México: Trillas.

LEÓN, T. Y RODRÍGUEZ, R. (2008). El efecto de la orientación vocacional en la elección de carrera. Revista Mexicana de Orientación Educativa, 5, 10-16. http:// pepsic.bvsalud.org/pdf/remo/v5n13/v5n13a04.pdf

LOBATO, C. (2002). Psicologíay asesoramiento vocacional. Revista de Psicodidáctica, 13, 117-130. https://dialnet.unirioja.es/servlet/articulo?codigo=866429

LOMPREY, G. (2012). Actitud y aptitud del docente de matemáticas con respecto a las TIC y su relación con el logro académico en la prueba enlace intermedia en los estudiantes de tercer año de secundaria [Resumen de tesis doctoral, Universidad de Montemorelos]. http://dspace.biblioteca.um.edu.mx/xmlui/ bitstream/handle/20.500.11972/535/Tesis\%20G\%c3\%agrald\%20Lomprey. pdf? sequence=1\&isAllowed $=y$

LÓPEZ, E. (2018). El perfil del docente competente de Humanidades y Ciencias Sociales en el bachillerato mexicano desde la perspectiva del alumno. https://doi. org/10.6018/j/350041

MARTíN, M. (2015). Optimización del proceso de selección para incrementar la eficiencia terminal en los programas de posgrado [Tesis doctoral, Universidad de Granada, España]. https://dialnet.unirioja.es/servlet/tesis?codigo=56355

MORCHIO, I. (2009). Hacia una aproximación multidimensional e innovadora de los pilares de la orientación vocacional [Tesis doctoral, Universidad Nacional de Cuyo. Facultad de Filosofía y Letras]. https://bdigital.uncu.edu.ar/5305

MORALES, J. (2017). La orientación vocacional para la elección de carreras universitarias dirigida a estudiantes de educación media. Revista Internacional de Investigación y Formación Educativa, 39-76. https://www.ensj.edu.mx/ wp-content/uploads/2017/Og/La-orientaci\%C3\%B3n-vocacional-para-laelecci\%C3\%B3n.pdf

MUNGARRO, G. (2007). Motivos yelección de carrera [Tesis de maestría, Universidad de Sonora, México]. http://wwww.mie.uson.mx/tesis/mungarro_2007.pdf

MUNGARRO, G., ZAYAS, F. (2009). Elección de carrera docente. X Congreso Nacional de Investigación Educativa. http://www.comie.org.mx/congreso/ memoriaelectronica/v10/pdf/area_tematica_16/ponencias/0254-F.pdf

OSIPOW, S. (1976). Teorias sobre la elección de carrera. México: Trillas. 
PANQUEBA, M. Y MESA, C. (2014). Orientación vocacional y profesional a estudiantes de grado once de la institución educativa Silvino Rodriguez, sede Jaime Rook de la ciudad de Tunja [Tesis de licenciatura, Universidad Nacional Abierta y a Distancia UNAD]. https://repository.unad.edu.co/bitstream/ handle/10596/2757/1053606458.pdf?sequence=3\&isAllowed=y

PARSONS, F. (1909). Choosing a vocation [Elegir una vocación]. Londres: Gay y Hancock.

REAL ACADEMIA Española (2019). Aptitud. https://www.rae.es/dpd/aptitud

ROJAS CAIRAMPOMA, M. (2005). Tipos de investigación científica: una simplificación de la complicada incoherente nomenclatura y clasificación. Revista Electrónica de veterinaria, 6, 1-14. https://www.redalyc.org/pdf/636/63638739004.pdf

RUPERTI, E., GÓMEZ, L. Y PALMA, M. (2020). Características vocacionales y comportamiento social de la Generación Z. Caso El Triunfo, Ecuador. Maskana, 11(1), 26-32. https://dialnet.unirioja.es/servlet/articulo?codigo=7509923

SÁNCHEZ, E. (2003). La vocación entre los aspirantes a maestro. Educación XX $X_{1}$, 6. 203-222. https://idus.us.es/xmlui/bitstream/handle/11441/17087/file_1. pdf? sequence $=1$

SANTOS, M. Y PORTO, A. (2002). Género, valores y orientación vocacional en un contexto universitario para la salud, reto de nuestro tiempo. Educación XX1, 5 , 19-34. http://www.redalyc.org/pdf/706/70600503.pdf

SANTIAGO, P., MCGREGOR, I., NUSCHE, D., RAVELA, P. Y TOLEDO, D. (2012). Revisiones de la OCDE sobre la Evaluación en Educación. Secretaría de Educación Pública. http://www.oecd.org/education/school/RevisionesoCDE-evaluacion-educacion-Mexico.pdf

SECRETARÍA DE EDUCACIÓN Pública (2020). Construye T: Guía del personal directivo y docente para la construcción de la nueva normalidad.https://WWW. construye-t.org. $m x /$ nueva-normalidad/docs/guia_docentes.pdf?v=1

SCHILLING, A., GAJARDO, K. Y ALALUF, L. (2019). Construcción de intereses vocacionales de estudiantes que participan de un programa para continuar estudios de pedagogía. Formación universitaria, 12(5), 91-100. https://dx.doi. org/10.4067/S0718-50062019000500091

STABILE ET AL. (2017). Aspectos personales, sociales y contextuales, en la elección de carrera de psicología, reconocidos por ingresantes 2016. Anuario de investigaciones de la Facultad de Psicología. Universidad Nacional de Córdoba, 3(1), 228-237. https://revistas.psi.unc.edu.ar/index.php/aifp/article/ view/18102/18504

SÚPER, D. Y BOHN, M. (1973). Psicología Ocupacional. México: CECSA.

Vargas, R. (2019). Proyecto de vida y su relación con valores, auto-concepto y apoyo social percibido en estudiantes de secundaria. Psicolnnova, 3(1), 17-31. https://www.unibe.ac.cr/ojs/index.php/psicoinnova/article/view/25/25

VILLEGAS, M. (2009). Cuestionario de Intereses y Aptitudes de Luis Herrera y Montes. México: UNAM. 